PharmacoEconomics \& Outcomes News 869, p31 - 9 Jan 2021

\title{
Telemedicine improves drug adherence and reduces OOP costs
}

Telemedicine appears to improve drug adherence and reduce out-of-pocket (OOP) costs in low-income patients with rheumatic and musculoskeletal diseases (RMDs) in India, according to findings of a study published in Rheumatology.

This cross-sectional study conducted at a tertiary hospital in India in April 2020 investigated the effects of telemedicine consultations on disease status, socioeconomic status and the economic impact of COVID-19 in 373 patients with RMDs who were not receiving disease-modifying antirheumatic drugs. Direct medical costs, direct nonmedical costs and indirect costs were assessed from the patient's perspective.

Prior to the COVID-19 pandemic, the median per capita income was $\$ 26^{*}$ per month per family and the cost of illness was $\$ 22$ per month.

During the pandemic, $72.7 \%$ of patients' families experienced loss in income (median $\$ 65$ per month), and daily financial needs were not met in $32.7 \%$ of families. Patients were significantly less likely to discontinue prescription drugs if financial needs of their family were met (odds ratio [OR] 0.38; 95\% Cl 0.239, 0.598) or their education level was secondary school level or higher (OR 0.442; 95\% Cl 0.260,0.752).

It was estimated that $77 \%$ of patients could be successfully switched to telemedicine.

"During times of crisis, patients from poorer strata of society and lower educational background are likely to abruptly stop medications. Switching to a telemedicine-based hybrid model is likely to improve drug adherence with substantial savings on loss of pay and out of pocket expenditure," concluded the investigators. "The main strength of this study was that the teleconsultations were done without incurring any additional expenditure and within a time frame of 3 weeks after the lockdown was announced, reflecting the financial and logistic ease of implementation," they said.

* 2020 US dollars

Kavadichanda C, et al. Tele-rheumatology for overcoming socioeconomic barriers to healthcare in resource constrained settings: lessons from COVID-19 pandemic. Rheumatology : 7 Dec 2020. Available from: URL: http://doi.org/10.1093/rheumatology/keaa791 